\title{
APPLICATION PERSPECTIVE OF 2D+SCALE DIMENSION
}

\author{
Hairi Karim and Alias Abdul Rahman \\ 3D GIS Research Group, Department of Geoinformatic, Faculty of Geoinformatic and Real Estate, \\ Universiti Teknologi Malaysia Johor Bahru, Johor, Malaysia.
}

KEY WORDS: scale dimension, current scale application, potential scale application, data structure and 3D vario-scale model.

\begin{abstract}
:
Different applications or users need different abstraction of spatial models, dimensionalities and specification of their datasets due to variations of required analysis and output. Various approaches, data models and data structures are now available to support most current application models in Geographic Information System (GIS). One of the focuses trend in GIS multi-dimensional research community is the implementation of scale dimension with spatial datasets to suit various scale application needs. In this paper, 2D spatial datasets that been scaled up as the third dimension are addressed as 2D+scale (or 3D-scale) dimension. Nowadays, various data structures, data models, approaches, schemas, and formats have been proposed as the best approaches to support variety of applications and dimensionality in 3D topology. However, only a few of them considers the element of scale as their targeted dimension. As the scale dimension is concerned, the implementation approach can be either multi-scale or vario-scale (with any available data structures and formats) depending on application requirements (topology, semantic and function). This paper attempts to discuss on the current and new potential applications which positively could be integrated upon 3D-scale dimension approach. The previous and current works on scale dimension as well as the requirements to be preserved for any given applications, implementation issues and future potential applications forms the major discussion of this paper.
\end{abstract}

\section{INTRODUCTION}

GIS with scale dimension has gained popularity in recent years due to the demands from users and applications. GIS research community is now moving forward for integrating a highly formal definition of geo-data (Oosterom and Stoter, 2010) and focusing on designing efficient framework for temporal and scale dimension. This research focuses on scale implementation within a recently develop Dual Half Edge (DHE) data structure (Boguslawski, 2011). As for introduction, scale dimension can be implemented either by multi-scale or vario-scale approach.

Similarly with other dimension, the implementations of scale dimension also require the selection of a data model and data structure; especially for vario-scale approach. Contrary for 3Dscale in multi-scale approach, there is no standard or minimum topological element required for data structure selection during the implementation process. Thus, less analytical assessments and query functions are expected without implementation of data structure for multi-scale approach. We have successfully implemented the vario-scale concept in the DHE structure using a small experimental land cover dataset. This 3D-scale DHE model able to support topology (navigation between objects of the same and different level of details (LoD), local modification, minimum redundancy of dataset and workload, capability to store attribute, topology and geometry within a single data structure.

Section 2 explains the previous works on scale dimension including data structure and the 3D-scale DHE model. Section 3 and 4 will describe on scale implementation in current GIS applications and potential of future applications; requirements, issues, best approaches and benefits offered. The last section of this paper will be the discussion and remark from this study.

\section{THE CONCEPT OF SCALE AND DATA STRUCTURE}

This section is divided into two main parts; scale dimension and the DHE data structure.

\subsection{Scale Dimension}

There is a wide definition on the third dimensional model, but normally, 3D refers to the height of 2D spatial data (to represent 3D space). However, a 2D geometry of spatial dimension with a non-spatial data (e.g. scale or time) is also valid concept in defining the $3^{\text {rd }}$ dimension (Oosterom, 2005).

Previous research papers has shown that most successful implementation of scale dimension started by introducing multiscale concepts in storing line simplification such as Multi-scale Line tree (Jones and Abraham, 1986), Arc-tree (Günther, 1988), and Binary Line Generalization (BLG) (Oosterom, 1990). The scale dimension implementation can be categorized either in multi-scale or vario-scale approach.

\subsubsection{Multi-scale Approach}

Nearly all previous works implemented different generalization methods in order to produce less-detailed LoDs from the higher ones. Hiding less important details or/and reducing the data storage volume are among of available options for an efficient 3D analysis and visualization (Baig et al., 2011).

The current implementation frameworks of multi-scale integration with 2D spatial data are either using the generalization techniques or storing the individual level of detail data into separate databases (Figure 1). On the other hand, 3D spatial GIS data normally uses a generalization 
technique (aggregation, simplification and other approaches) or a set of pre-defined level of details (LoDs, see Figure 2) which are topologically unconnected to represent scale dimension or connected by IDs at the same level (Ohari et al, 2015).

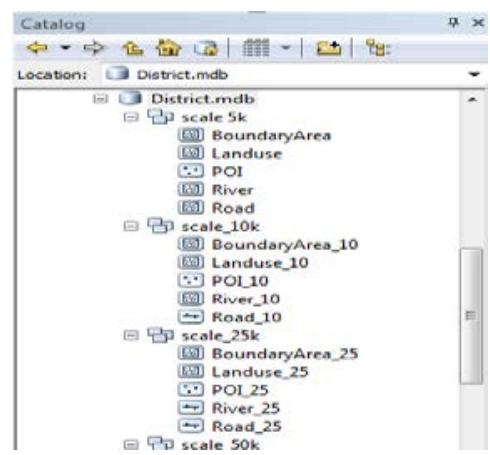

Figure 1. A 2D redundancy problem in multi-scale approach

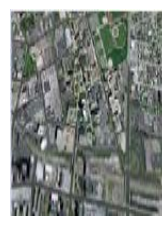

LoD0

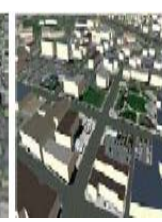

LoD1

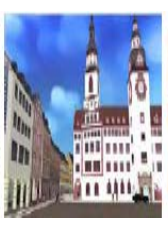

LoD2

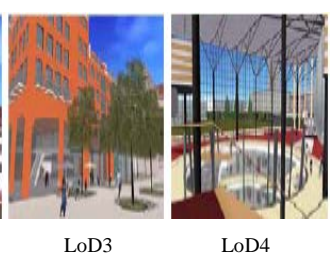

LoD3

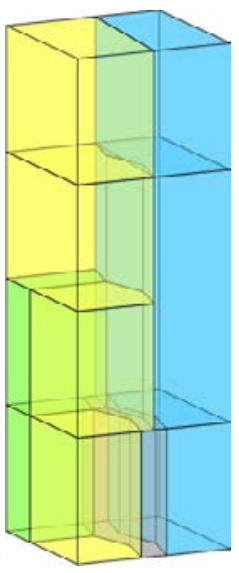

b)

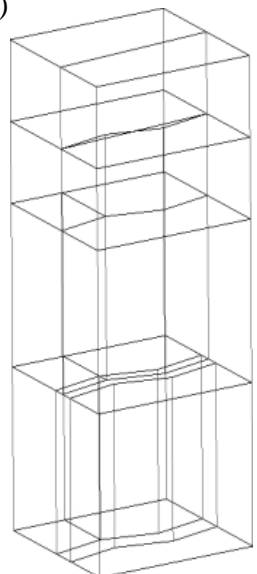

c)

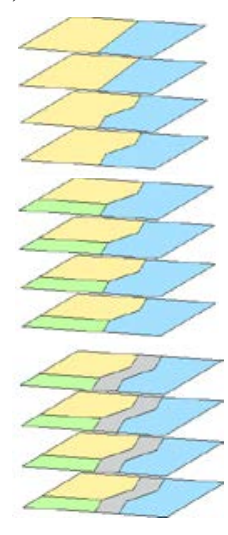

Figure 4. A classic tGAP model (Meijers, 2011); a) Classic Space Scale Cube (SSC) model, b) wireframe model, c) slicing planes for several LoDs

\subsection{Dual Half Edge Data Structure}

A data structure is define as a specialized format or approach for organizing and storing of digital geographic dataset in a particular spatial data model. It refers to the approach on how to encapsulate the geometry, topology and semantic information of the GIS data systematically for the purpose of analyzing the world with its objects and phenomena within digital model (Boguslawski, 2011). Ledoux (2006) also noted that different data structures can be implemented in a single data model.

It is impossible and not practical to design a general-purpose data structure to work with all kind of situations or applications (Meijers, 2011). Thus, the selection of an appropriate data structure to be implemented inside a data model is chosen based on the targeted applications and dimensionality structure (Boguslawski, 2011; Gold, 2005).

The DHE uses two structures; the dual and the primal which presented as entities connected to form a graph (Boguslawski, 2011). The dual structure conforms to the rules of the 3D Poincaré duality (Boguslawski, 2011). A cell in primal structure presented as a dual vertex; a face as a dual edge, an edge as a face and lastly, a vertex is presented as a cell. Figure 5 illustrates the concept of 3D Poincaré duality in DHE structure. (Meijers, 2011). The vario-scale approach is an extension of tGAP structure which uses hierarchical tree structure.

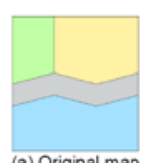

(a) Original map
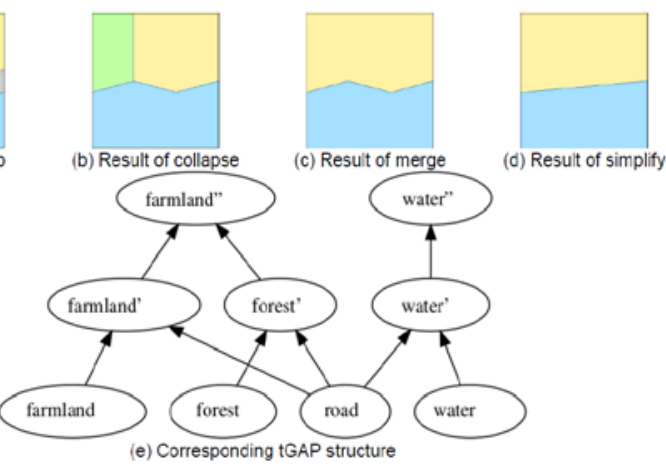

Figure 3. The process of generalization and hierarchical tGAP structure (Oosterom and Meijers, 2011).

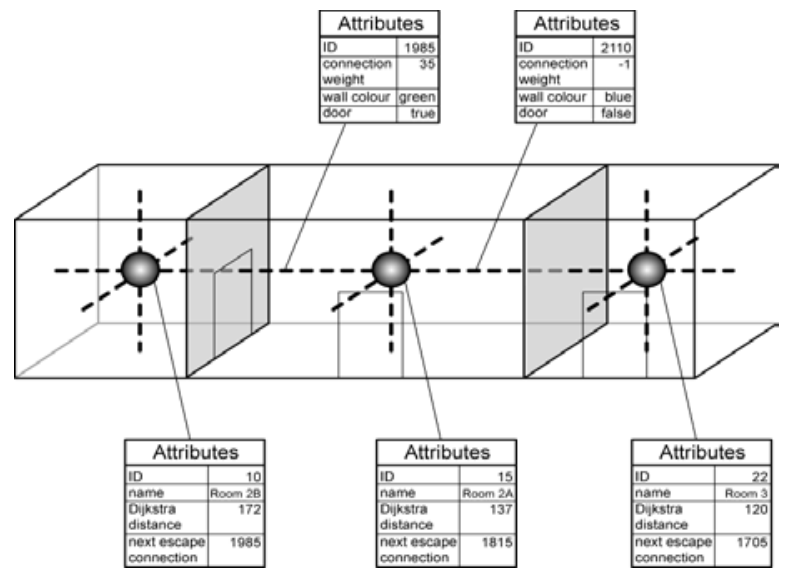

Figure 5. Poincaré duality with the primal space to support 
The International Archives of the Photogrammetry, Remote Sensing and Spatial Information Sciences, Volume XLII-4/W1, 2016 International Conference on Geomatic and Geospatial Technology (GGT) 2016, 3-5 October 2016, Kuala Lumpur, Malaysia

topology and the geometry of 3D cell (Boguslawski, 2011).

Navigation in DHE data structure is possible because all of halfedges in the model are connected via pointers. The concept of half-edge was applied to represent edges in the model in whereby all edges are split into two directed halves. The navigation is performed from half edge to half edge - each half edge consists of directed half edge with dual half edge.

\subsubsection{The DHE Vario-scale Concept}

The DHE data structure is capable to support the geometry, topology and attribute and with additional scale dimension (let's say, $l$ ) to replace $\mathrm{Z}$ axis. So, we will have a series of $\mathrm{XY} l_{0}$, $\mathrm{XYl}_{1}, \mathrm{XY}_{2}, . . \mathrm{XY} l_{n}$ (where $n$ is the number of object and $\mathrm{XY}$ is the original coordinate of an object without any geometry generalization/changes). The concept of DHE vario-scale model is illustrated in Figure 6 which uses the basic of hierarchical tree structure using the sequence of its dual vertexes and edges.

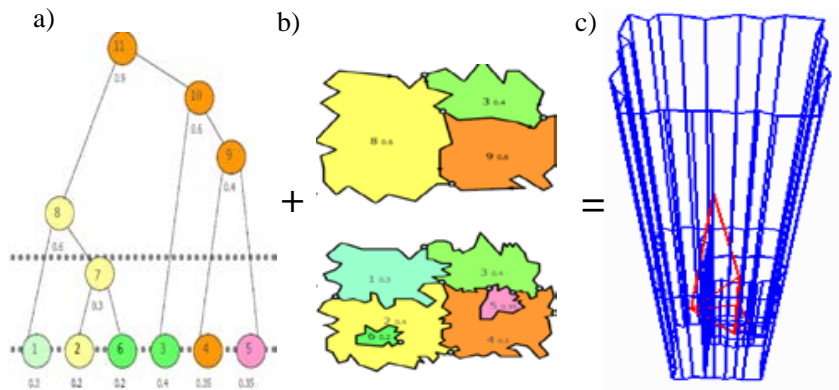

Figure 6. The idea on implementing the DHE vario-scale data model in a single model.

a)

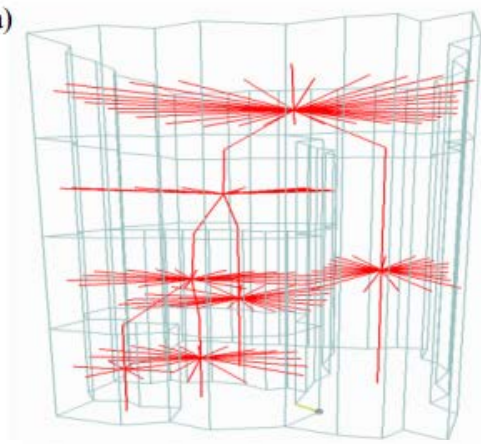

c)

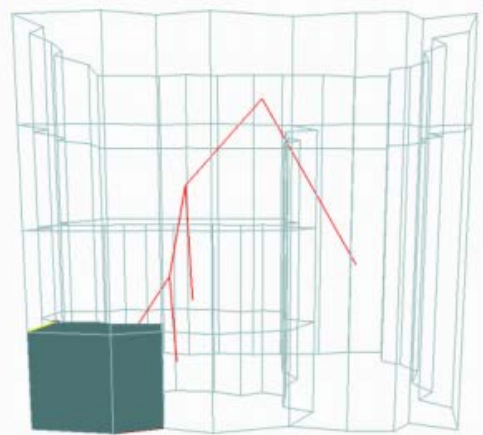

\subsubsection{The DHE Vario-Scale: Experimental Model:}

The implementation of 3D DHE vario-scale model starts with the basic and topological cleaned 2D dataset (validated without geometry and topological error), followed by 2D-to-3D DHE generalization technique and ended with slicing approach to acquire various of LODs as final result. Figure 7 shows a sample 2D dataset used for the experimental small dataset. Details implementation processes of generating 2D-to-3D with vario-scale DHE model are described in Karim et al. (2016).

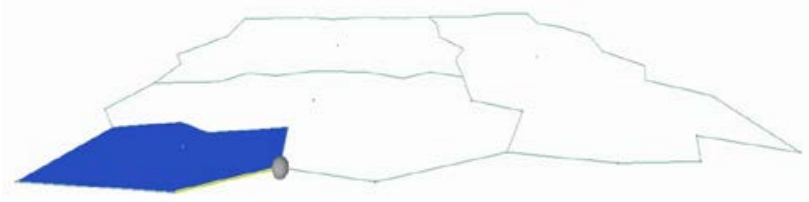

Figure 7. An example of 2D scale dataset for the experimental.

Basically, generalization of the 2D-to-3D DHE vario-scale incorporate three extrusion techniques (either by point, polyline or polygon-based extrusion), 'merge by face' and 'topology simplification' to generate a valid output. These extrusion techniques are quite complex (case sensitive) since it also involves the topological extrusion (topological creation and validation) once geometry of an object is being extruded. Figure 9 shows the overall processes of converting 2D dataset (Figure 7) to $3 \mathrm{D}(\mathrm{XYI})$ via 2D-to-3D DHE vario-scale generalization method technique. The result shows the generalization process by utilizing the normal extrusion technique (extrusion by polygon), merge by face and topology simplification. b)

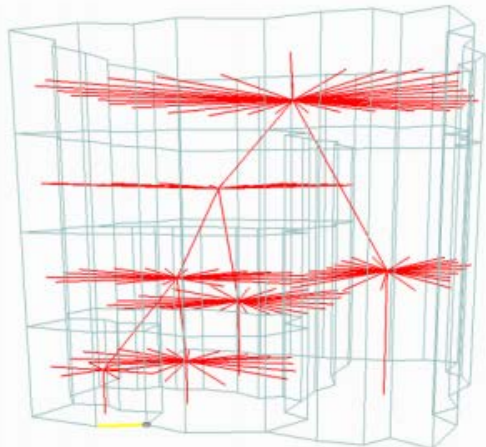

d)

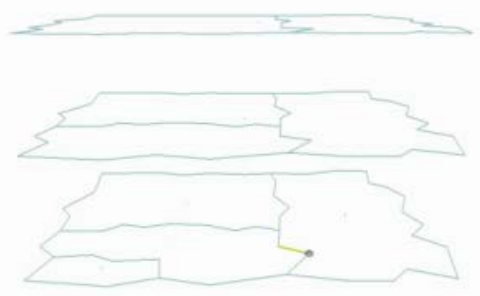

Figure 9. Overall process of generalization; a) extrusion technique, b) Merge by Face operator, c) Simplified version of the dual (for navigation and query across scale level), d) corresponding LoDs (Karim at el., 2016) 


\section{EXISTING APPLICATIONS FOR SCALE DIMENSION}

There are a few existing applications which use the scale as the third dimension after spatial 2D dataset. Most of the current implemented applications use multi-scale approach to fit their working fields. Some of them are topographical data, land cover and land use.

\subsection{Topographic data}

Conventionally, topographic maps represented both natural and man-made features using various approach, including a series of map sheets to represent at particular map scale (Figure 10). A modern and online topographic map however uses quantitative representation of relief, usually using contour lines and without man-made features. The implementation of online topographic map usually without national standard (mostly focuses on international cartographic and thematic approach), started from most details layer, hiding some features when it being zoom out and finally being replaced by the less detailed layer (refers to Figure 11a, 11b, 11c).

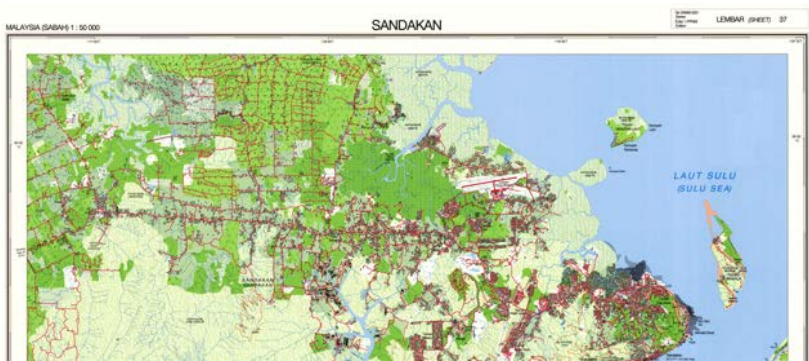

Figure 10. An example of standard 1:50,000 topographic map.

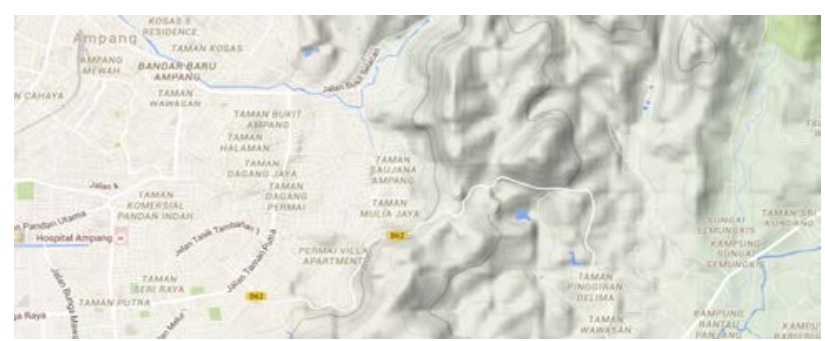

Figure 11a. An example of most detailed topographic map.

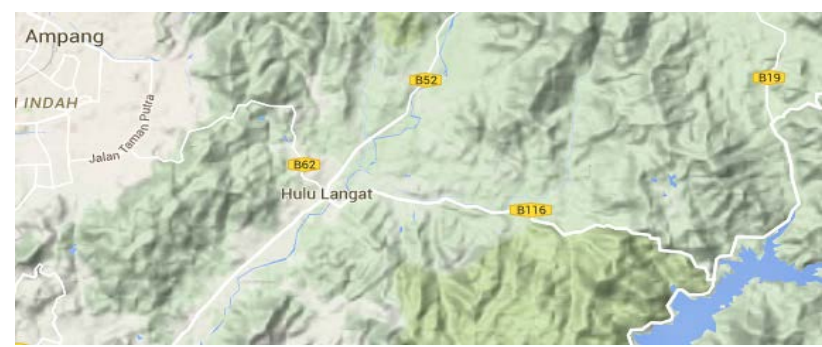

Figure 11b. Concealation of information (road and contour).

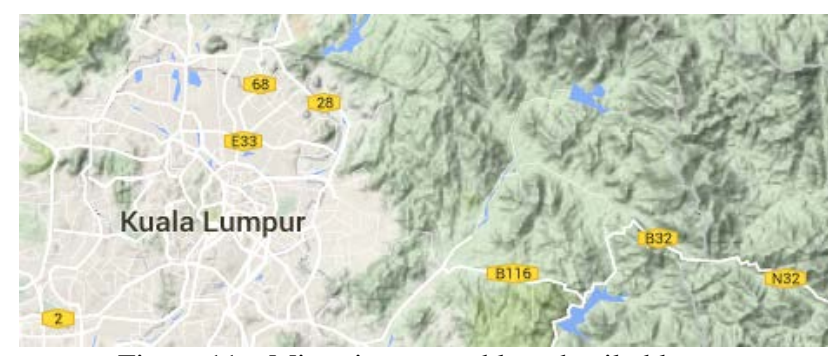

Figure 11c. Migration toward less detailed layer.

\subsection{Land cover data}

The purpose of land cover map is to serve a detailed vegetation and land use patterns especially for development and ecological classification. According to United State Geological Survey (USGS), a national program called 'GAP Land Cover' dataset uses approximately 590 land cover classes in 3 different level of details (USGS, 2015) . Figure 12 shows an example of 13 land cover classes for Hawai'i Island, US.

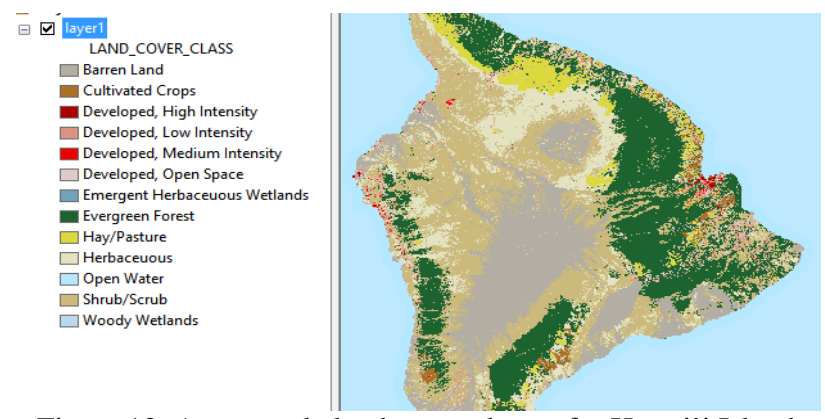

Figure 12. An example land cover classes for Hawai’i Island.

Land cover datasets normally being used as a reference to develop models related to distribution of habitat or national's vertebrate species (NLCD, 2014). It is very important for decision makers (gavernonce of the particular area), the planners, researchers and private interests such as in managing the national protected forest and others (Figure 13). Information on land covers also served as basic creterions for biodiversity, habitat loss and climate changes for simulation analysis.

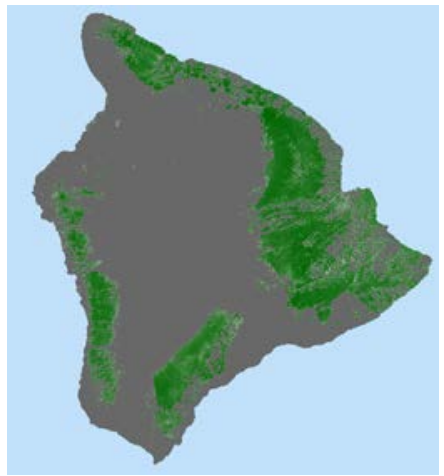

Figure 12. Forest land cover for protection (NLCD, 2014).

Although the land cover datasets management are mostly done without scale dimension implementation (such in different scales or LoDs), but, this paper considers it has been successfully implemented via vario-scale approach. Oosterom and Meijers (2011) used a small sample of The Netherlands land cover datasets to implement the concept of vario-scale approach in 3D SSC model (2D spatial + scale). Recently, Huang et. al (2016) implemented vario-scale approach in online 
The International Archives of the Photogrammetry, Remote Sensing and Spatial Information Sciences, Volume XLII-4/W1, 2016 International Conference on Geomatic and Geospatial Technology (GGT) 2016, 3-5 October 2016, Kuala Lumpur, Malaysia

European ATKIS (Authoritative Topographic-Cartographic Information System) in designing engineering web maps.

\subsection{Land use data}

The application of generalization techniques has been proven successfully by research of Monmier (1983). He applies the generalization technique that able to extract land cover and soil information from raster dataset. Recently, Dimov at el. (2014) has successfully implemented an automated generalization technique using GIS-based requirement to the Valencia land use dataset. The result (Figure 13) is then being compared with Information System of Land Cover in Spain (SIOSE) datasets for validation purposes.

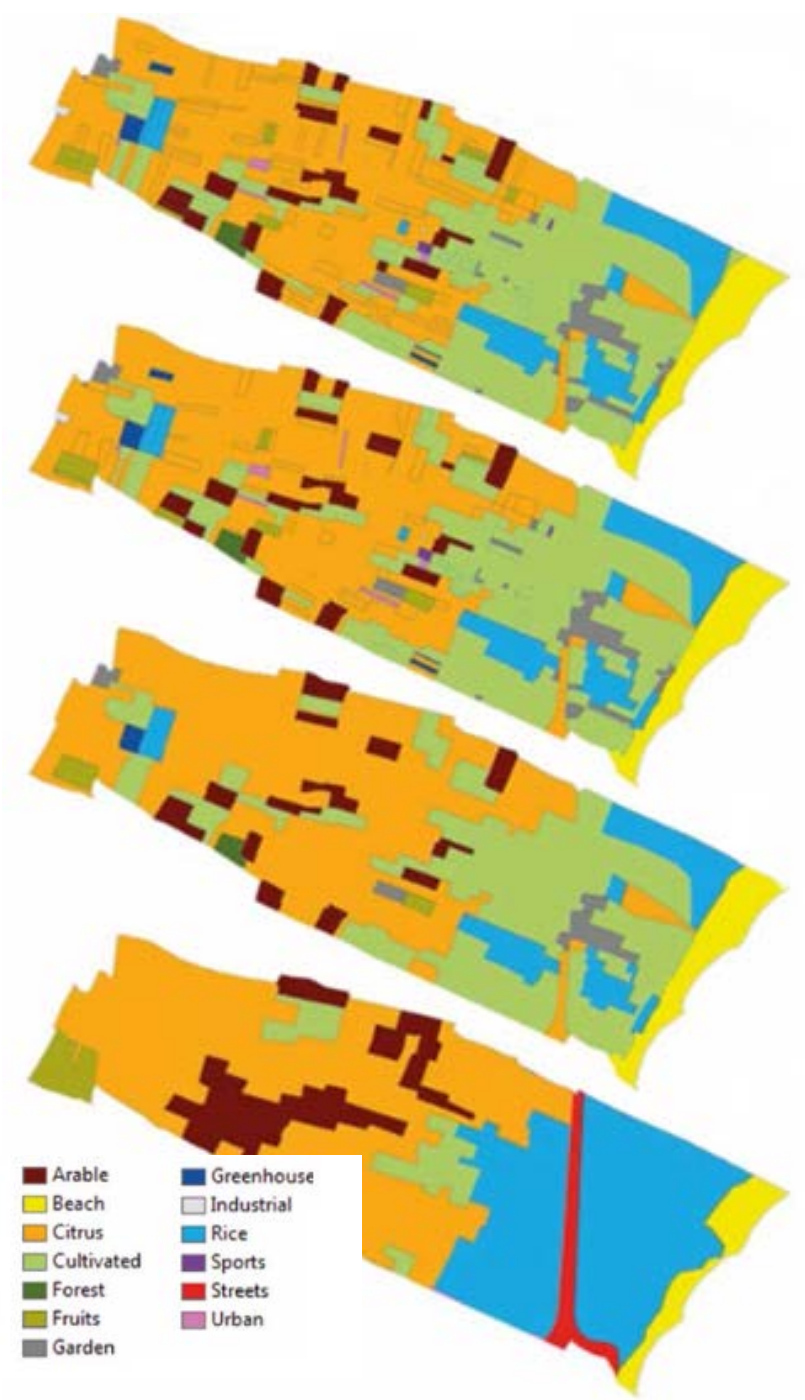

Figure 13. A series of automated generalization technique on land use by Dimov at el. (2014)

\section{POTENTIAL FUTURE APPLICATIONS}

In this section, we will explore some issues on scale modelling and the significant requirement of scale implementation for future potential applications.

\subsection{Soil Maps}

Hawai'i Soil Atlas Geoportal is one of the best example on the requirement of scale dimension in managing soil dataset (refers to series of Figure 14). A number of 297 different soils and land cover types, providing details information on topographic location, climate, and attributes describing soil properties (e.g. water retention, fertility, acidity or alkalinity, organic matter, physical structure and others). Numerical data that essential for controlling plant nutrients and properties related to soil productivity also recorded for diagnosis of nutrient sufficiency or deficiency.

In Figure 14, a single soil feature (polygon unit) is selected (red colour) to illustrate the current implementation in soil maps application without scale dimension approach. Geometry and attribute generalization of the soil layer is one of the promising approaches to have a better visualization in a large scale map.
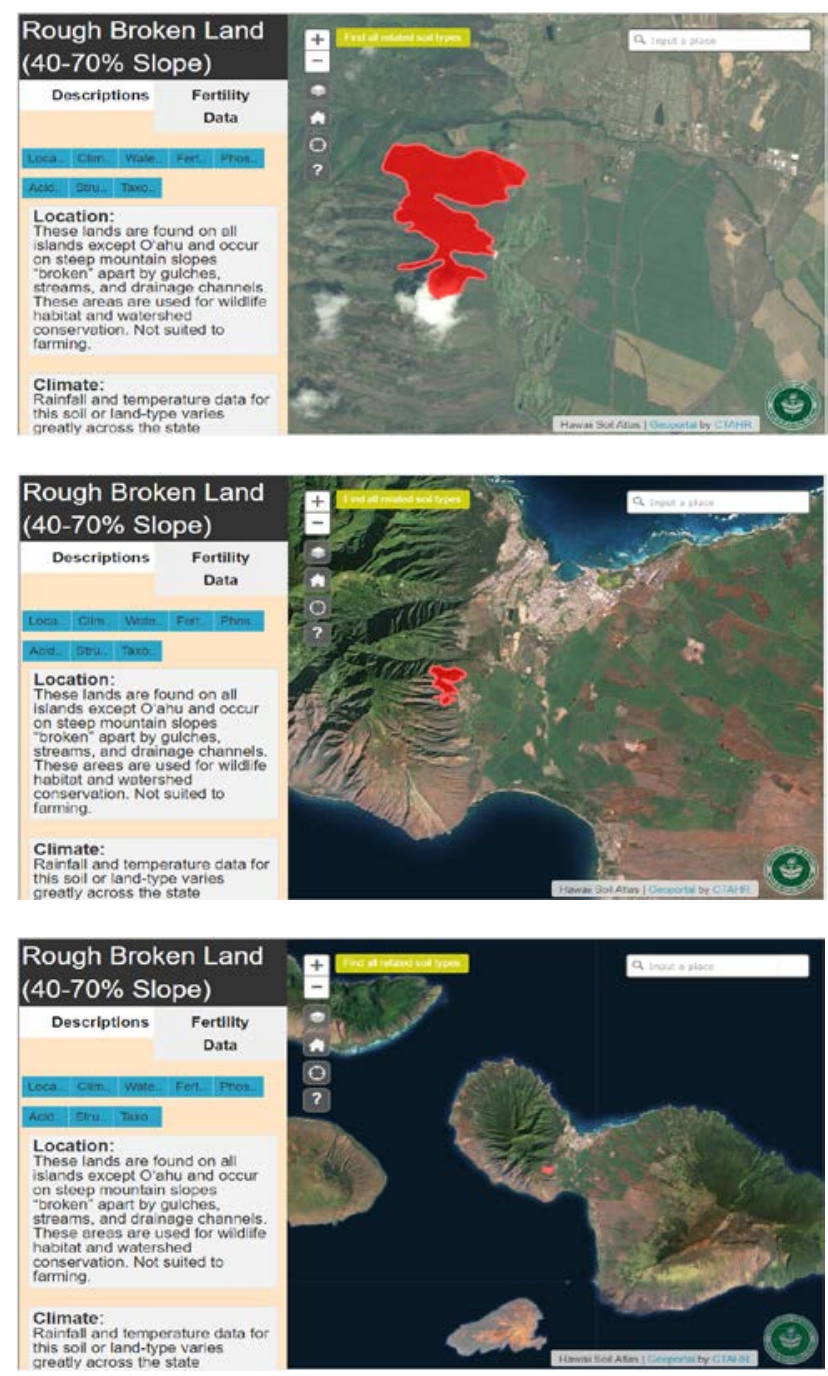


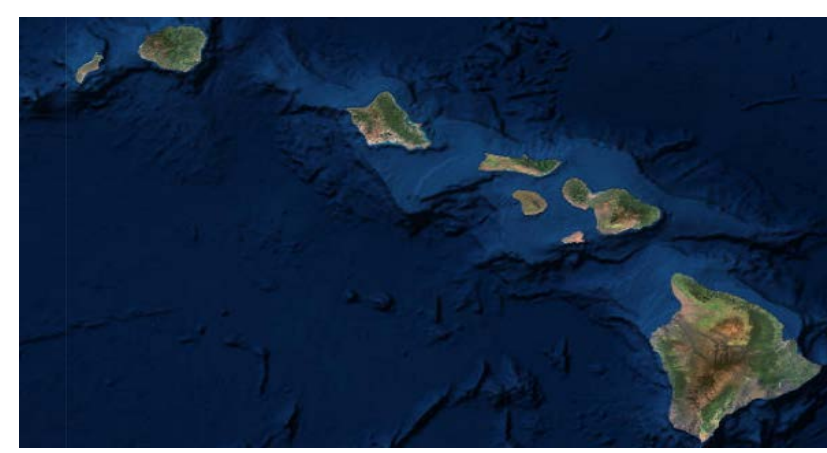

Figure 14. A series of different map scales using same object geometry and attribute (CTAHR, 2014).

\subsection{Density Maps}

Density map is a method used to describe the differences of quantitative measurement (e.g. population, event and others) and distributions over geographic landscape. Thematic maps, dotted or line (line segment for traffic condition) or population density, pie or bar chart maps and hot-spot maps (e.g. crime events) are some examples of density map presentation. Scale dimension becoming useful to model the density map datasets when it comes to illustrate difference scale ratios (different value over bigger area) in a particular application. Figure 15 illustrate population density (census 2010) from a scale of a city, country and the world population which different in number per kilometre square $\left(\mathrm{km}^{2}\right)$; represented in different colours.

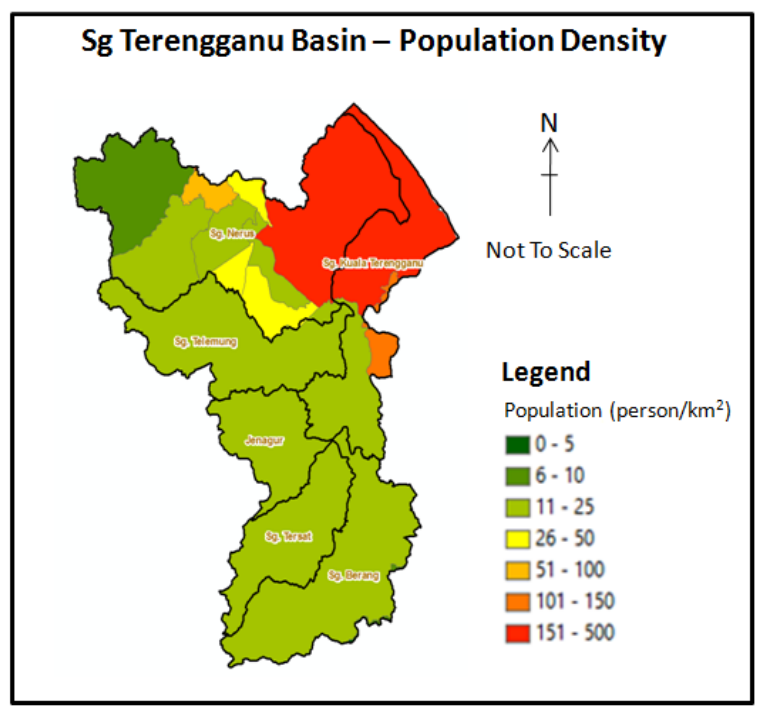

Figure 15a. A large scale population density map of Kuala Terengganu (Capital city for Terengganu State, Malaysia).

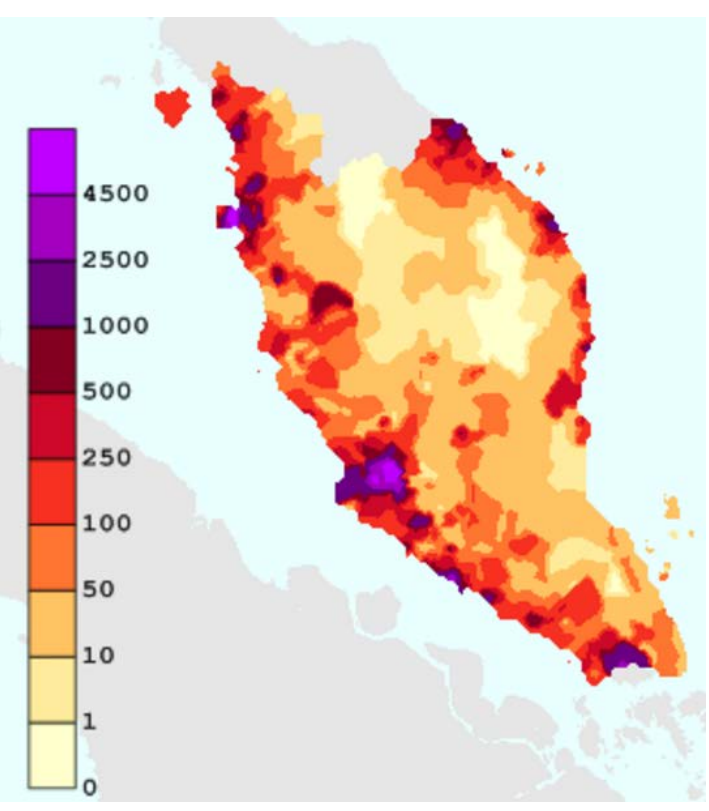

Figure 15b. Medium scale of population density for Peninsular Malaysia Wikimedia (2016).

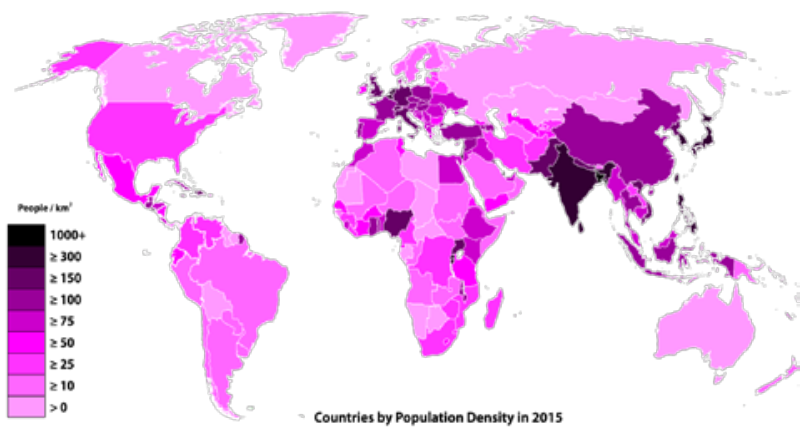

Figure 15b. Large scale of world population density.

\subsection{D As-Built Drawing (Complex Building)}

In some cases, scale dimension is also needed to simplify the spatial layers especially for a crowded area (e.g. point of interest (POI), megamall and tourism area). Accurate spatial information regarding the location, services and goods are essential in guiding tourist or those who are not familiar with the area. Getting lost in crowded area may affect the tourism in term of safety, time consuming and deception tendency from irresponsible shop owner.

A proper guideline which is not too detailed and not too simple (depending on the maps user) is needed to ensure the tourist or visitor go to the right targeted location without any obstacle and getting out from crowd efficiently. Thus, a smart 2D floor plan should be designed to show the user only straight forward spatial information. For example, a clustered food stalls or food court can be generalized into two or three famous restaurants. Figure 16a shows the $2^{\text {nd }}$ floor of mid-valley shopping mall as an example of crowded POI in Kuala Lumpur area. Figure 16b shows the initial model of 2D floor plan for $2^{\text {nd }}$ floor of MidValley Megamall in the DHE structure. 


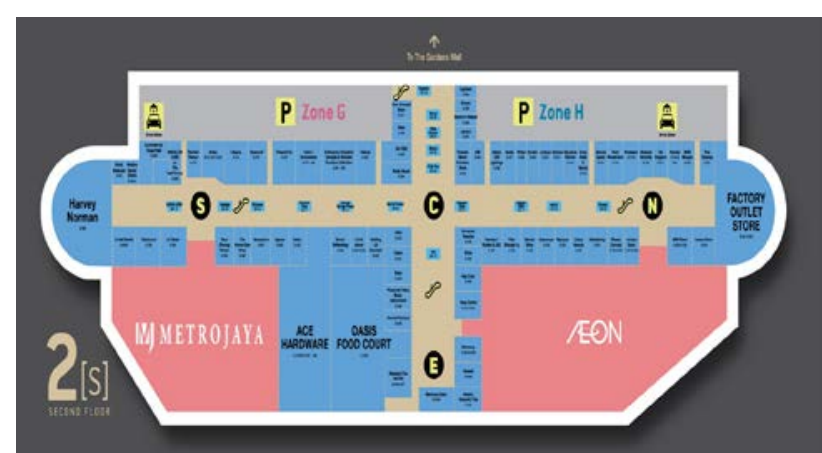

Figure 16a. $2^{\text {nd }}$ floor (more than 95 stores) out of five shopping mall floors at Mid-Valley Megamall.

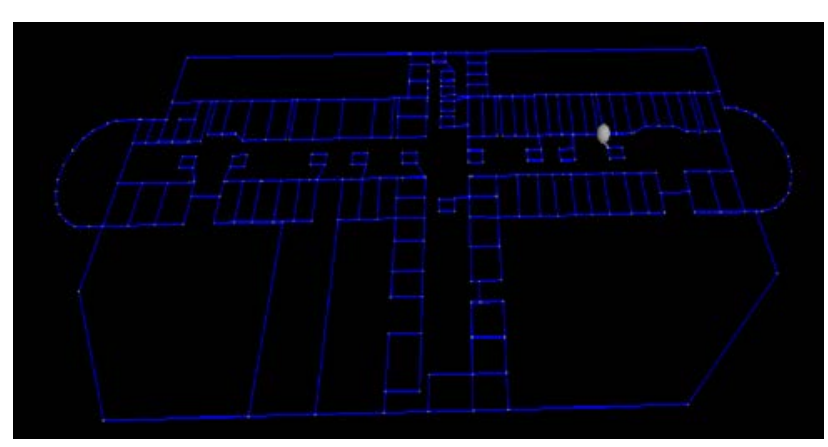

Figure 16b. Initial 2D DHE model of the $2^{\text {nd }}$ floor.

\subsection{D Cadastral Maps}

The available generalization technique normally is not suitable to represent 2D cadastral map. Multi-scale approach basically is not a best practise for this application since land parcel details is a sensitive geometry shape - related to the ownership of land parcel. However, there is a demand on securing the cadastral land parcel map (freehold) by the authority to share the information about the neighbour's parcel details. Figure 17a and $17 \mathrm{~b}$ show a selected land parcel in which undergo 10 varioscale generalization levels on its neighbour parcels.

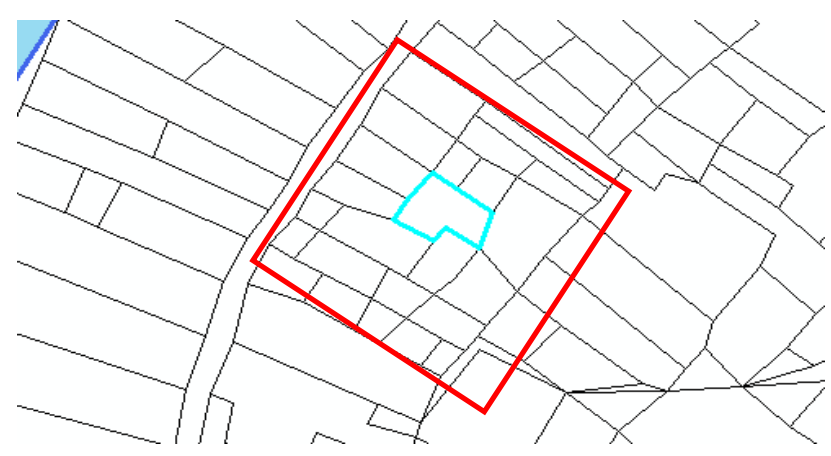

Figure 17a. Original land parcel

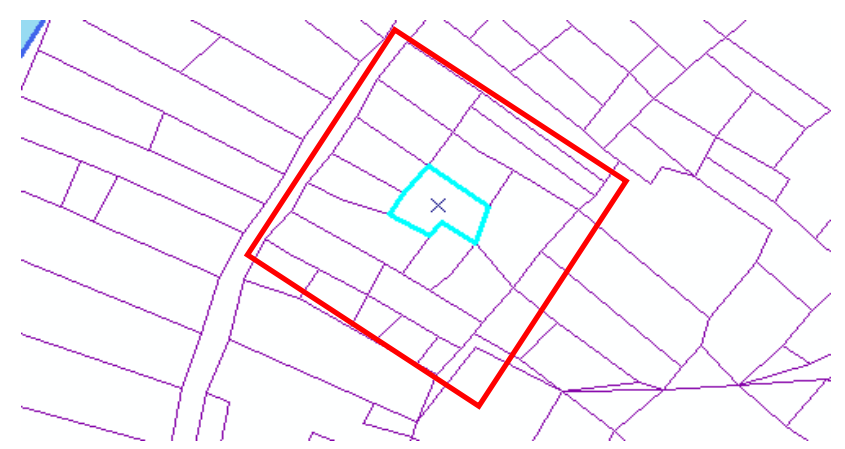

Figure 17b. Land parcel after neighbour’s generalization.

The figures (17a and 17b) show implementation of the DHE vario-scale generalization will not affect the outer boundary of the neighbour's objects. In other words, the processes of merging two objects maintain the geometry and attribute the selected object and its neighbours that really fits the requirement for cadastral application.

\section{CONCLUSION}

There are two possible approaches in implementing scale as a new dimension for spatial 2D, they are - multi-scale and varioscale. The DHE vario-scale has introduced a generalization technique that capable to navigate topologically into successor and predecessor, primal and dual primitives. Each generalization process maintains the outer boundary of the two corresponding objects.

This paper discussed the potential of implementing scale dimension into several existing and potential scale-applications. Some requirements are needed in order to maintain the originality of the data in each application or dataset while implementing the scale dimension. The study on these requirements for each application is necessary to develop a weighted parameter for the scale implementation. For example, the soil with very good nutrient should be highlighted in the next scale level (not selected for generalization) as compared to less nutrient soil in agriculture application.

The DHE vario-scale model and 2D to 3D DHE generalization technique seem to be another possible implementation approach for spatial scaling application. The discussed potential applications (less review available in these subjects) are just some initial idea from author's perspective to expand the application domain for scale implementation.

We attempt to focus on implementation of the DHE vario-scale model into one of the discussed potential application using its weighted parameters for our future works. We believe that the 3D DHE vario-scale model with the proposed generalization technique will be beneficial toward spatial scale application.

\section{REFERENCES}

Baig, S.U., Hassan, M.I. and Rahman, A.A., (2011). Automatic Generalization of 3D Building Models - A Review, 10th International Symposium \& Exhibition on Geoinformation (ISG 2011). ISPRS, Shah Alam Convention Centre(SACC), Malaysia.

Boguslawski, P., (2011). Modelling and Analysing 3D Building Interiors with the Dual Half-Edge Data Structure, University of Glamorgan, UK, 1-134 pp.

CTAHR, (2014). Hawai'i Soil Atlas , College of Tropical Agriculture and Human Resources (CTAHR) Geoportal, University of Hawai'i, Accessed on July 2016. http://gis.ctahr.hawaii.edu/SoilAtlas.

Dimov D., Palomar J.M., Ruiz A.L. (2014), Automated Generalization of Land-Use Data with GIS-Based Programming 
Gold, C. M. (2005). Data structures for dynamic and multidimensional GIS. In Proceedings of the 4th ISPRS Workshop on Dynamic and Multi-dimensional GIS (pp. 36-41).

Günther, O., (1988). Efficient Structure for Geometric Data Management. Number 337 in Lecture Notes in Computer Science. Springer-Verlag, Berlin. Springer Book.

Huang, H., Meijers, M., Šuba. R., Oosterom, P.V., (2016). Engineering web maps with gradual content zoom based on streaming vector data . ISPRS Journal of Photogrammetry and Remote Sensing 114 (2016) 274-293.

Jones, C.B. and Abraham, I.M., (1986). Design Considerations for a Scale Dependent Cartographic Database. 384-398.

Karim, H., Rahman, A.A., and Boguslawski, P., (2016). Generalization Technique for 3D-Scale DHE Data Model, GeoAdvances 2016, Istanbul. (In Progress).

| Ledoux, H., 2006. Modelling Three-dimensional Fields in Geoscience with the Voronoi Diagram and its Dual. PhD Thesis. Certificate of Research, University of Glamorgan.

Monmonier M., (1983). Raster-mode Generalization for Land Use and Land Cover Maps. Cartographica The International Journal for Geographic Information and Geovisualization 20(4):65-91 - October 1983.

Meijers, M., (2011). Variable-Scale Geo-Information, PhD Thesis. Technische Universiteit Delft, Netherlands.

NLCD, (2014). National Land Cover Database, land cover Hawai'i (Data Basin Dataset). ArcGIS archived portal. Accessed on July 2016.https://www.arcgis.com/home/item.html?id=1e970d07f49f 4c7cb996f9dc65ca9f39

Ohari, K.A., Ledoux, H., Biljecki, F., and Stoter, J., (2015). Modeling a 3D City Model and Its Levels of Detail as a True 4D Model. ISPRS International Journal of Geo-Information 2015. DOI:10.3390/ijgi4031055

Oosterom, P.v., (1990). Reactive Data Structure for Geographic Information Systems, PhD Thesis. Leiden University.

Oosterom, P.V., (2005). Variable-scale Topological Data Structures Suitable for Progressive Data Transfer: The GAPface Tree and GAP-edge Forest. Cartography and Geographic Information Science(32): 331-346.

Oosterom, Peter van, \& Meijers, Martijn. (2011). Towards a True Vario-scale Structure Supporting Smooth-zoom. Paper presented at the 14th ICA/ISPRS Workshop on Generalization and Multiple Representation, Paris.

Oosterom, P.V. and Stoter, J., (2010). 5D Data Modelling: Full Integration of 2D/3D Space, Time and Scale Dimensions, 6th international conference on Geo- graphic information science. Springer- Verlag, Berlin, Heidelberg, pp. pages 310-324.

Sester, M., (2007). 3D Visualization and Generalization. Institude of Cartography and Geoinformatics. Leibniz University of Hannover, Online presentation. http://www.ifp.uni-stuttgart.de/phowo/2007/ presentations/320sester.pdf (pp. 10)

USGS, (2015). National Gap Analysis Program (GAP) | Land Cover Data Portal, United State Geological Survey (USGS). Accessed on July 2016. http://gapanalysis.usgs.gov/gaplandcover/data/

Wolfram, (2016). World Population Density Plotted with A Logarithmic Color Scale, Computation Meet Knowledge, Accessed on July 2016. http://community.wolfram.com/groups/$/ \mathrm{m} / \mathrm{t} / 854271$

Wikimedia (2016). Map of Malaysia Population Density 2010. Accessed on July 2016. https://commons.wikimedia.org/wiki/ File:Malaysia_population_density_2010b.png

Wikipedia (2016). List of countries by population density. Accessed July2016.https://simple.wikipedia.org/wiki/List_of_countries_b y_population_density\#/media/File:Countries_by_Population _Density_in_2015.svg 